\title{
Proposal of driving system with the function of connection and separation of cars for the realization of automatic driving society
}

\author{
Yu Nakagawa ${ }^{a,},{ }^{*}$ Naoyuki Ishizima ${ }^{a}$, Makoto Miyauchi $^{b}$, Seiichi Serikawa ${ }^{a}$ \\ ${ }^{a}$ Kyushu Institute of Technology, 1-1 Sensui-cho Tobata-ku Kitakyushu Fukuoka-ken, 804-8550, Japan \\ ${ }^{b}$ National Institute of Technology, Kitakyushu College \\ *Corresponding Author: nakagawa.yu616@ mail.kyutech.jp
}

\begin{abstract}
This paper aims to propose development of the next generation car robot after a fully automatic driving society has been established. It shows a robot that can be automatically coupled and separated from the next generation car robot. We believe that many people can be freely transported to different places by being able to connect and separate cars and cars. In addition, by communicating with smartphones and communicating between cars, we aim to develop a system that enables us to go from where we are now to the destination without using the effort. At present, we have achieved the development of car robots that can do line tracing for robot development.
\end{abstract}

Keywords: automatic driving, connect, separate

\section{Introduction}

Toward fully automated driving (level 5), research is currently being conducted at companies and universities. As far back as the development of automatic driving technology, research and development has been carried out all the time though the activity fell down on the way, starting from the research and development of automatic driving by computer vision technology around 1975. As an automation technology in automobiles, a safe driving support system has been put into practical use, in which a control system intervenes in the driving operation of a driver and supports safe driving of the driver when a danger of an accident approaches due to drift of drivers or dozing driving It is widespread. In order to realize automatic operation at the level of automation level 3 or higher, significant technological innovation is required for the performance of sensing technology and information processing technology currently in practical use in safe driving support systems. Technology development aiming at realizing level 3 or more automatic operation in Japan, the USA, and Europe is under way.

If the research continues with this trend, society is expected that decades later, hundreds of years later a complete operation automation (level 5) was established. Once complete operation automation is established, you do not need a driver's seat. By connecting multiple cars, people can come and go by attaching a door like a train in front of and behind the car. Furthermore, you can call a car at any time by communicating with a car using a smartphone, and you can drive to the destination. Currently, there is a concatenated bus, this bus can carry many people at once and can go to your destination. However, although this person can pass through the connection part of this bus, it can not be automatically coupled and separated. As an example, Volvo has the bus of $30 \mathrm{~m}$, which is the longest in the world, for Bus Rapid Transit System (BRT) in Rio de Janeiro, Brazil. There are two connecting parts of this bus, and it is composed of three cars. Also the bus can carry 300 passengers. However, if it is a huge bus, it is necessary to make a big round when turning, its driving is difficult. Therefore, the car robot we develop is small, we combine and separate, focusing on carrying a lot of people to the destination. The image of the articulated bus is shown in Fig. 1. 


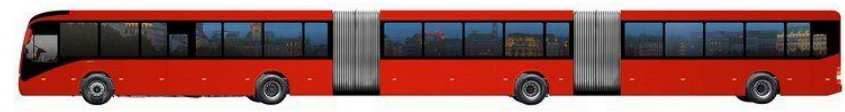

Fig. 1. Connection bus

By train, you can carry a lot of people at once, but there are many people far from the station to home. So I will ride my research wherever I am, and make a car robot that will allow me to reach my destination without getting off the car even once. The following things can be pointed out as the things that can not be done currently with concatenated buses.

(1) Automatic connection and separation

(2) Driving with only one car

(3) Multiple communication

What we do in this study is as follows.

(1) Multiple vehicles that can run independently, combine and separate.

(2) In the case of three or more vehicles, separate the vehicles in the middle and the rear, and then let them run their own.

(3) Communicate with smartphones and cars so that you can call the car at any time.

The mechanism of coupling and separation is shown in the following Fig. 2 to Fig. 4. Fig. 2 shows a coupled state, Fig. 3 shows a separated state, and Fig. 4 shows a coupled state.

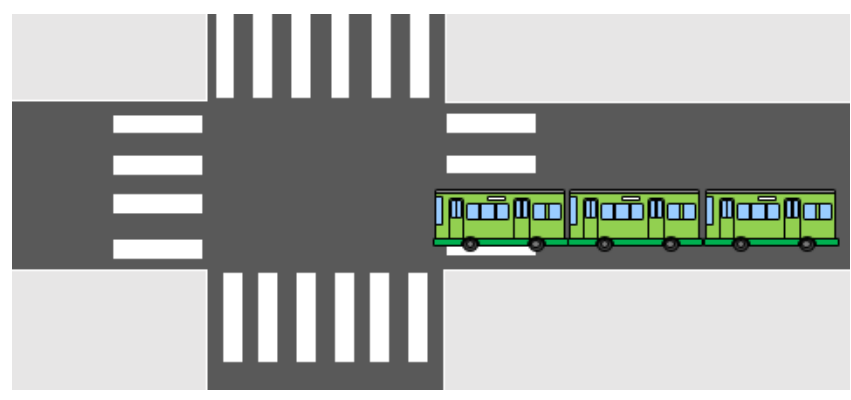

Fig. 2. Coupled state

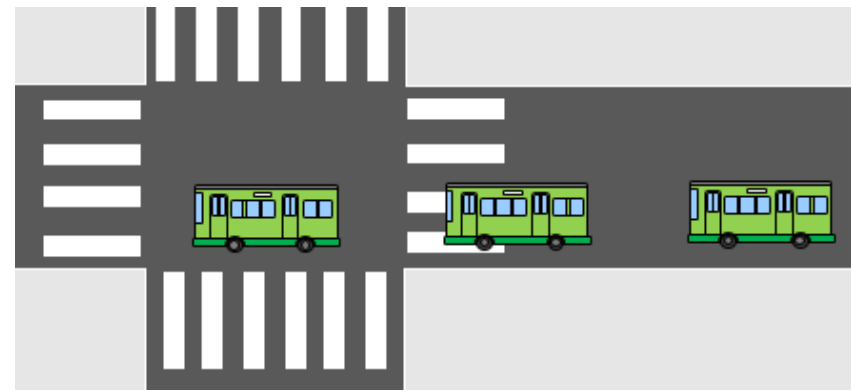

Fig. 3. Separated state

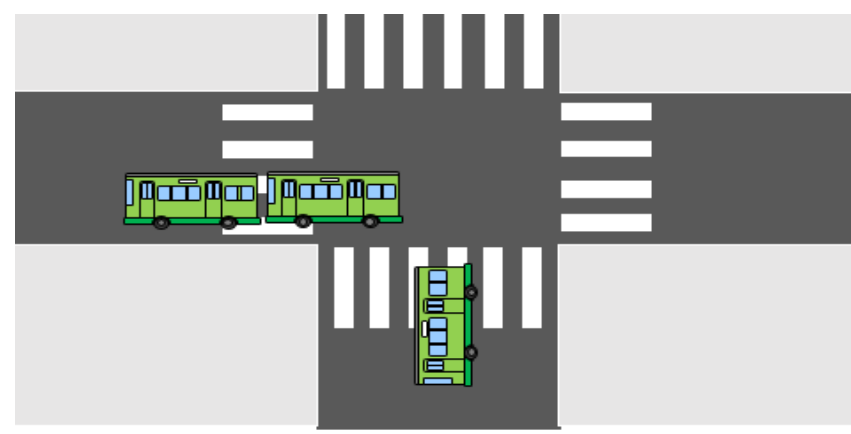

Fig. 4. connected state

Therefore, in this paper, we propose a traveling system that can coalesce and separate cars for complete operation automation society.

\section{Existing research}

The Adaptive Cuise Control (ACC) System which has been researched and developed and commercialized in the past is a follow-up type using the following distance. The ACC system can follow but can not combine. The main purpose of the ACC system is to reduce operation load. Regarding this point as well, it is assumed that no driver is present, so no burden on driving is required. Also, since we have no driver's seat, we believe we can combine smoothly by combining after following up.

Regarding the communication, there is an ITS safe driving support radio system, and information from the inter-vehicle communication system that supports safe driving by wireless communication in which vehicles exchange information with each other, such as intersections with poor visibility and infrastructure (traffic signal information, regulation information, Pedestrian information, etc.) from the dew side machine to the vehicle, via roadside radio communication via radio waves. Inter-vehicle communication is a system that obtains information (position, speed, vehicle control information, etc.) of 
surrounding vehicles by wireless communication between vehicles, and performs safe driving support to the driver as necessary. At intersections with bad visibility, cars transmit and receive their own vehicle information such as the position and speed of each other by radio, and if there is a danger of encounter collision, warn the driver to prevent a collision accident. This inter-vehicle communication is for informing the driver, but we move the car itself to the destination by communicating between the cars. The destination here is the place where someone called the car and the destination of the people riding in the car.

Our research uses Model B of Raspberry Pi 3 for communication. Raspberry $\mathrm{Pi}$ is a hardware called "one board microcomputer" which can be used by installing power supply and SD card storage instead of installing the internal hard disk and so on. Raspberry $\mathrm{Pi}$ is used in various things all over the world because performance is inferior to the latest spec computer, but not only built-in, but also various things can be made. Because it has HDMI output, USB port, LAN port, it is suitable for IoT because it can connect directly with the device body (display, keyboard, etc.). There are three main things that can be done using Raspberry Pi.

\section{(1) Incorporating the equipment body}

What is being used the most is embedded. It can be used as embedded Linux because it can load a Linux OS. When using embedded Linux, a microcomputer board becomes necessary. Therefore, it is common to use Raspberry Pi which is small and inexpensive. Examples of use are robots, electronic equipment, medical equipment, etc.

(2) IoT to connect with the main body of the device and the Internet

Because it has HDMI output, USB port, LAN port, you can directly connect to the main body of the device, build network environment - It is possible to carry out numerical calculation and control on the virtual server. Therefore, it is indispensable to IoT. Especially it plays an active part in sensors. Adjust temperature by refrigerator, microwave oven, air conditioner etc. with temperature sensor. Controlled by aggregating vibrations and shocks of cars and machinery with an acceleration sensor. Sensing sensor cooperates with surveillance cameras and intercom cameras to detect abnormalities. As I said, I am good at coordinating with things.

\section{(3) Application building}

You can build Raspberry $\mathrm{Pi}$ as a web application server. It is because it has HDMI output, USB port, LAN port and cost reduction can be expected. Conventional servers cost about 60 watts. By using Raspberry Pi it can hold down to $3.5 \mathrm{w}$, so it can save a lot of power. Moreover, since it can supply power with UPS battery, it can be used for more than 2 hours even in case of power outage.

\section{An image of Raspberry Pi 3 is shown in Fig. 5.}

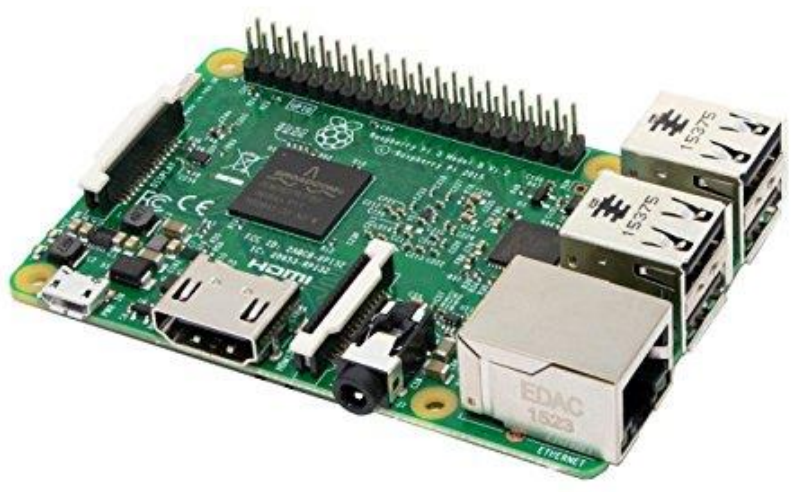

Fig. 5. Raspberry Pi 3

\section{System proposal for connection and separation}

\subsection{Traveling system}

The shape of the car robot is a rectangular box type, and in connection, it uses a motor and connecting parts. Make your own self so that you can drive, combine, separate and communicate car robots. Complete automation of cars (level 5) Considering society, assuming that you can run automatically anywhere, do it in line trace form. Because we do not need a driver's seat, we create a program using Rasberry Pi for a box-type car robot. At first, we create a line trace like a cross and write a program to make it run fine on the line. Two or three car robots come closer to each other and shift to coupling / separation when they stop.

\subsection{Connection and separation}

Motors and connecting parts are attached to the tip of a plurality of car robots, and when the car stops, join. When joining, move the motor of one car and connect the connecting part to the front car. In separation, when the car robot is bent at an intersection or the like, the motor is rotated in the reverse direction to the coupled state, 
whereby the connection is released and separated.

\subsection{Recognition}

By installing a camera on a car robot and incorporating Raspberry $\mathrm{Pi}$, it is a method of recognizing by using image processing. Detect the LED attached to the car with the camera and process it with Raspberry Pi.

\section{Conclusions}

At present, we have achieved the development of car robots that can do line tracing using ARM microcomputer for robot development.

In the future, we will create a car robot fitted with Raspberry Pi and separate and join on the cross line while running the line. Communication is added, communication from smartphone and cars are communicated, and we develop a system where we can come wherever we are.

\section{References}

(1) Yoshinori Yamamura, Youji Seto and Masao Nagai : "Study of vehicle group stable ACC using inter-vehicle communication", Journal of the Japan Society of Mechanical Engineers (Part C), Vo73, No. 726, pp. 379, 2007

(2) Yoshihiro Suda and Keiji Aoki : "Current activities and some issues on the development of automated driving", Journal of information Processing and Management Vo57, No. 11, pp. 809-817, 2015

(3) Sadayuki Tsugawa: "Automated Driving of Automobiles:Its Benefits and Issues”, IPSJ SIG Technical Report, Vo2009-MBL-51, No.9, pp. 1-8, 2009

(4) Keiji Aoki : "Current activities and some issues on the development of automated driving", Journal of information Processing and Management, Vo60, No. 4, pp. 229-239, 2017 